\title{
COMUNICACIÓN
}

\section{RECUPERACIÓN DE PRADERAS DEGRADADAS POR MEDIO DE CLAUSURAS TEMPORALES}

\author{
Ramiro Farfán L. 1, Felipe San Martín H.2 y Adrián Durant O.3
}

\section{Abstrat}

The impact of eliminating grazing on the recovery of degraded high elevation wet and dry Andean puna pastures is documented. In each ecosystem, areas of $625 \mathrm{~m}^{2}$ were fenced and 20 alpacas were grazed for 14 days to provide natural fertilisation prior to closure. After two years without grazing at the wet puna test site at 4,100 meters above sea level in Marampaqui, (Quispicanchis, Ocongate, Cusco), biomass increased by $308 \%$, plant cover grew by $16 \%$ and carrying capacity improved by $400 \%$ from 1.9 to 7.6 animals per hectare. Pasture composition altered with the appearance of 13 new plant species, an $8 \%$ decrease in the desirable Alchemilla pinnata, a $71 \%$ increase in the undesirable Calamagrostis vicunarum and a $50 \%$ decrease in the undesirable Aciachne pulvinata species, among others. At the dry puna test site at 4,300 meters above sea level in the community of Titiri, (Mariscal Nieto, Moquegua), biomass increased by $27 \%$, plant cover grew between 28 and $69 \%$, and carrying capacity improved by $40 \%$ from 0.5 to 0.7 animals per hectare. A total of 6 desirable new species appeared and production of the desirable Alchemilla pinnata increased by $950 \%$. Among the undesirable species, Calamagrostis vicunarum increased by $31 \%$ and Astragalus sp. disappeared.

Key words: Pasture recovery, native pastures, carrying capacity.

Palabras clave: Recuperación de praderas, pastizales, praderas naturales, capacidad de carga

Son diversas las causas que explican el origen del proceso de degradación de las praderas naturales en el sector altoandino del sur del Perú. Se puede asumir que este proceso empieza cuando los camélidos domésticos son replegados hacia las partes al-

I Estación Experimental Maranganí IVITA - FMV - UNMSM.

2 Laboratorio de Bioquímica y Alimentación Animal IVITA - FMV - UNMSM.

E.mail:d170032@unmsm.edu.pe

3 Práctica privada tas de la cordillera, generando una mayor presión de pastoreo; a la introducción de nuevas especies de animales domésticos como vacunos, ovinos y equinos, que tienen una mayor demanda alimenticia y; el aumento de áreas para cultivos agrícolas reduciendo las praderas nativas generalmente de mayor productividad.

Por esta razón se planteó el presente estudio con el fin de evaluar alternativas que permitan recuperar las praderas o detener el proceso de deterioro a través de la clausura y fertilización con heces (Cuadro 1). 
Se instalaron dos áreas de clausura de $625 \mathrm{~m}^{2}$ de extensión con malla ganadera de 9 hilos y postes de fierro, excluyendo este espacio del pastoreo normal. Al interior de estas clausuras se alojaron, a manera de dormidero temporal, 20 alpacas durante 14 días con fines de fertilización natural aprovechando el estiércol y la orina excretada por los animales. Como testigo se consideraron las áreas de pastizales adyacentes a las clausuras, las que tuvieron una presión de pastoreo normal con los animales del fundo.

Al momento de la instalación de las clausuras y durante dos años consecutivos se realizaron labores de evaluación continua de los siguientes indicado:

- Rendimiento de las especies botánicas elegidas como indicadoras (deseables, poco deseables y no deseables).

- Incremento o reducción de la cobertura vegetal.

- Composición botánica.

- Producción de biomasa en términos de forraje verde y materia seca, considerando solamente las especies forrajeras deseables y poco deseables.

- Capacidad de carga animal expresada en Unidades Animal Alpaca por hectárea año (UAA/ha/año).

- Desarrollo de especies palatables nuevas en las áreas clausaradas en relación a las áreas testigo.

Para obtener y procesar esta información se utilizó los métodos de Puntos de Contacto (Farfán y Durant, 1998) y Cuadrado de Corte (Flórez, 1993) usándose formularios pre-establecidos. Los pastos muestreados fueron secados al medio ambiente en sombra por 15 días. Los resultados fueron expresados en porcentaje de cambio comparando las áreas clausuradas en las áreas testigo.

El Cuadro 2 muestra los resultados de la evaluación de dos años (1997 y 1998) en puna húmeda. Se puede apreciar que la especie deseable indicadora Alchemilla pinnata desciende de $13 \%$ a $12 \%$. Este comportamiento posiblemente se deba a la falta de pastoreo y por estar localizada en una comunidad vegetal abierta (Flórez, et al.,1992). Además, en esta área se observa la aparición de otras especies que crecen con agresividad y generan una cierta competencia interespecífica que afecta el crecimiento de la A. pinnata. Por otro lado, se observa en el área clausurada un incremento en la frecuencia de Calamagrostis vicunarum, forrajera poco deseable y la aparición de 13 nuevas especies deseables y poco deseables no presentes en el área testigo. La especie Aciachne pulvinata, considerada no deseable para el pastoreo, desciende en su frecuencia.

En el área clausurada la cobertura vegetal se incrementa e igualmente la producción de biomasa, cuyo rendimiento final genera un aumento en la capacidad de carga de 1.9 a 7.6 UAA/ha/año.

Los resultados en puna seca se muestran en el Cuadro 3. Existe un incremento en la frecuencia de Alchemilla pinnata, forrajera deseable y altamente preferida por alpacas; en este caso la clausura permitió su pleno desarrollo en los dos años que duró el experimento. Aunque con menor velocidad la especie Calamagrostis vicunarum, poco deseable, incrementó su frecuencia y la especie Astragalus sp, no deseable, desapareció al final del experimento. También, se observó en área 
Cuadro 1. Zona ecológica, localización y altitud de las áreas clausuradas.

\begin{tabular}{llc}
\hline Zona ecológica & Localización & Altitud, m \\
\hline Puna húmeda & $\begin{array}{l}\text { Comunidad campesina de Marampaqui, } \\
\text { sector Tapamayo - Ocongate - Cusco. }\end{array}$ & 4,100 \\
Puna seca & $\begin{array}{l}\text { Comunidad campesina de Titiri - } \\
\text { Mariscal Nieto - Moquegua. }\end{array}$ & 4,300
\end{tabular}

Cuadro 2. Respuestas de praderas degradadas a clausuras temporales en puna húmeda en la Comunidad Campesina de Marampaqui.

\begin{tabular}{|c|c|c|c|c|}
\hline Índices & $\begin{array}{c}\text { Palata- } \\
\text { bilidad* }\end{array}$ & Testigo & Clausura & $\begin{array}{c}\text { Cambio } \\
\%\end{array}$ \\
\hline \multicolumn{5}{|l|}{ Especie indicadora, \% } \\
\hline Alchemilla pinnata & D & 13 & 12 & -8 \\
\hline Calamagrostis vicunarum & PD & 7 & 12 & 71 \\
\hline Aciachne pulvinata & ND & 8 & 4 & -50 \\
\hline Cobertura vegetal, $\%$ & & 85 & 99 & 16 \\
\hline Producción de biomasa, $\mathrm{kg} \mathrm{MS/ha}$ & $\mathrm{D}+\mathrm{PD}$ & 800 & 3260 & 308 \\
\hline Capacidad de carga, U.A.A./ha & & 1.9 & 7.6 & 300 \\
\hline \multicolumn{5}{|l|}{ Especies palatables nuevas, $\%$} \\
\hline Hordeum muticum & $\mathrm{D}$ & 0.0 & 4.0 & \\
\hline Trifolium repens & $\mathrm{D}$ & 0.0 & 3.5 & \\
\hline Paspalum pygmaeun & $\mathrm{D}$ & 0.0 & 3.0 & \\
\hline Bromus catharticus & $\mathrm{D}$ & 0.0 & 1.3 & \\
\hline Poa pratensis & $\mathrm{D}$ & 0.0 & 1.0 & \\
\hline Festuca dolichophylla & $\mathrm{D}$ & 0.0 & 1.0 & \\
\hline Bromus pitensis & $\mathrm{D}$ & 0.0 & 0.8 & \\
\hline Gnaphalium capitatum & $\mathrm{D}$ & 0.0 & 0.8 & \\
\hline Bromus unioloides & $\mathrm{D}$ & 0.0 & 0.5 & \\
\hline Hipochoeris sp & $\mathrm{D}$ & 0.0 & 0.5 & \\
\hline Muhlembergia ligularis & $\mathrm{D}$ & 0.0 & 0.3 & \\
\hline Calamagrostis curvula & PD & 0.0 & 1.3 & \\
\hline Stipa sp & PD & 0.0 & 0.3 & \\
\hline
\end{tabular}

* $\mathrm{D}=$ Deseable; $\mathrm{PD}=$ Poco deseable; $\mathrm{ND}=$ No deseable 
Cuadro 3. Respuesta de praderas degradadas a clausuras temporales en puna seca en la Comunidad campesina de Titiri.

\begin{tabular}{lcccc}
\hline Indices & $\begin{array}{c}\text { Palata- } \\
\text { bilidad* }\end{array}$ & Testigo & Clausura & $\begin{array}{c}\text { Cambio } \\
\%\end{array}$ \\
\hline Especie indicadora, \% & D & 2 & 21 & 950 \\
$\begin{array}{l}\text { Alchemilla pinnata } \\
\text { Calamagrostis vicunarum }\end{array}$ & PD & 13 & 17 & 31 \\
Astragalus sp. & ND & 4 & 0 & -100 \\
Cobertura vegetal, \% & & 28 & 69 & 146 \\
Producción de biomasa, kg MS/ha & D + PD & 220 & 280 & 27 \\
& & & & \\
Capacidad de carga, U.A.A./ha & & 0.5 & 0.7 & 40 \\
& & & & \\
Especies palatables nuevas, \% & & & & \\
Calamagrostis sp. & $\mathrm{D}$ & 0.0 & 5.3 & \\
Alchemilla erodifolia & $\mathrm{D}$ & 0.0 & 2.0 & \\
Geranium sessiliflorum & $\mathrm{D}$ & 0.0 & 1.8 & \\
Carex sp & $\mathrm{D}$ & 0.0 & 0.8 & \\
Trifolium repens & $\mathrm{D}$ & 0.0 & 0.5 & \\
Poa sp & $\mathrm{D}$ & 0.0 & 0.3 & \\
\hline
\end{tabular}

*D $=$ Deseable; $\mathrm{PD}=$ Poco deseable; $\mathrm{ND}=$ No deseable

clausurada un incremento y desarrollo de seis nuevas especies deseables y poco deseables, especies no presentes en el área testigo. La cobertura vegetal se incrementó de $28 \%$ en el área testigo a $69 \%$ en el área clausurada.

La producción de materia seca se incrementó en un $27 \%$ al final de los dos años de evaluación, favoreciendo la capacidad de carga en un $40 \%$.

En los dos ensayos se observaron aparición de diversas especies forrajeras palatables cuyo orígen se puede atribuir a la fertilización natural y la presencia de semillas no digeridas pero escarificadas encontradas en el estiércol de los animales que utilizaron las clausuras como dormideros.

Se concluye que en las áreas clausuradas se incrementa en frecuencia la especie deseable Alchemilla pinnata y poco deseable Calamagrostis vicunarum, mientras tanto, las especies no deseables como el Astrágalus sp y Aciachne pulvinata decrecen y en algunos casos desaparecen por efecto de la clausura y la competencia inter-específica entre especies.

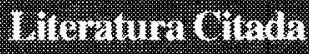

1. Farfán, R. y A. Durant. 1998. Manejo y Técnicas de Evaluación de Pastizales Altoandinos. Pub. Tec. FMVUNMSM- No 39. Lima Perú. 160 p. 
2. Flórez, A.; E. Malpartida y F. San Martín. 1992. Manual de forrajes para zonas áridas y semiáridas Andinas. 1ra. Ed. p:122. Red de Rumiantes Menores (RERUMEN). Lima, Perú.
3. Flórez, A. 1993. Producción y utilización de los pastizales altoandinos del Perú. Red de Pastizales Andinos (REPAAN). Quito-Ecuador. 202 p. 